\title{
e-Phaïstos
}

e-Phaïstos

Revue d'histoire des techniques / Journal of the history

of technology

V-1 2016 | 2018

Ville et technique

\section{L'ancienne France au quotidien. Vie et choses de la vie sous l'Ancien Régime, sous la direction de Michel Figeac}

\section{Cyril Lachèze}

\section{(2) OpenEdition}

\section{Journals}

Édition électronique

URL : http://journals.openedition.org/ephaistos/1152

DOI : 10.4000/ephaistos. 1152

ISSN : 2552-0741

Éditeur

IHMC - Institut d'histoire moderne et contemporaine (UMR 8066)

Référence électronique

Cyril Lachèze, "L'ancienne France au quotidien. Vie et choses de la vie sous l'Ancien Régime, sous la direction de Michel Figeac », e-Phaïstos [En ligne], V-1 2016 | 2018, mis en ligne le 09 novembre 2019, consulté le 24 septembre 2020. URL : http://journals.openedition.org/ephaistos/1152 ; DOI : https:// doi.org/10.4000/ephaistos. 1152

Ce document a été généré automatiquement le 24 septembre 2020.

Tous droits réservés 


\title{
L'ancienne France au quotidien. Vie et choses de la vie sous l'Ancien Régime, sous la direction de Michel Figeac
}

\author{
Cyril Lachèze
}

\section{RÉFÉRENCE}

Michel Figeac (dir.), L'ancienne France au quotidien. Vie et choses de la vie sous l'Ancien Régime, Paris, Armand Colin, 2007, 590p.

1 Malgré son titre, qui pourrait de prime abord évoquer un ouvrage de vulgarisation, il s'agit là d'un dictionnaire encyclopédique remarquable sur l'histoire de la culture matérielle de l'époque moderne. Cette publication, appelée par Michel Figeac lui-même «Dictionnaire d'histoire matérielle" ne porte donc pas, fondamentalement, sur l'histoire des techniques. Toutefois, tout matériel manufacturé supposant la mise en œuvre de procédés techniques pour sa création, celle-ci y est au final omniprésente. L'équipe de vingt-cinq auteurs, rattachés à plus d'une douzaine d'établissements de recherche différents, permet de couvrir un vaste champ de spécialités. Ce sont au total plus de deux centaines d'articles, souvent développés sur deux ou trois pages chacun, classés par ordre alphabétique, qui couvrent un très large éventail d'aspects de la société en lien avec le matériel : les articles portent aussi bien sur des objets au sens propre que sur des modes de vie, cadres d'habitation ou encore pratique artisanales.

2 L'avant-propos de cinq pages, rédigé par Michel Figeac, seule partie du livre qui ne soit pas consacrée aux articles en eux-mêmes, présente de manière claire la raison d'être de cet ouvrage. Celui-ci s'inscrit très explicitement dans la lignée des études déjà menées en histoire sur la culture matérielle moderne, en particulier les multiples travaux de Daniel Roche. L'étude des inventaires après décès, devenue monnaie courante dans les 
années 1990, produit une bonne connaissance au moins quantitative des objets composant les maisonnées. La multiplication des études de cas, pour elles-mêmes ou comme sous-ensembles d'études d'histoire sociale, ont permis de mettre en évidence l'importance du rôle de la culture matérielle dans l'affirmation des statuts sociaux, les décalages considérables qu'elle connaît entre les différents lieux ou composantes sociales, ou encore l'importance des outils dans la réussite sociale des artisans et propriétaires terriens. Le croisement des inventaires avec d'autres types de sources, par exemple les livres de raison ou encore les annonces publicitaires dans la presse, a permis d'aborder des phénomènes matériels plus complexes, tels que les effets de modes et la naissance de la société de consommation. Le but de L'ancienne France au quotidien consiste à regrouper ces différentes approches, parfois assez nouvelles, pour les faire converger dans une somme regroupant l'ensemble des acquis issus de différents mouvements historiens, voire de différentes disciplines. Deux niveaux d'analyse sont mis en évidence dans cette optique. Le premier est celui de la description de la réalité matérielle en elle-même; Michel Figeac mentionne ici la nécessité de travailler en liaison avec les historiens des arts décoratifs aptes à analyser les objets conservés dans les musées ; aucune autre spécialité n'est mentionnée, ce sur quoi nous reviendrons par la suite. Le second niveau d'analyse est social, avec la nécessité de replacer la culture matérielle dans son contexte, et notamment dans la dynamique des divers groupes composant une société en évolution. S'y ajoute la prise en compte, bien amenée et explicitée, de la technique, qui nous intéresse particulièrement ici, et de l'environnement.

3 Trois types de sujets d'articles apportent des éléments au point de vue technique. Le premier type, le plus fourni, correspond aux objets en tant que tels, comme «Bateaux et navires", "Bouteilles» ou "Pain». Le second regroupe des articles concernant plutôt des artisanats pris dans leur ensemble: "Métallurgie et sidérurgie », ou encore «Facture et lutherie ». Enfin, quelques articles abordent des thèmes plus généraux, par exemple la notion d'« Atelier » ou de «Boutique». Dans tous les cas, les articles suivent une structure proche, cohérente avec celle annoncée dans l'avant-propos, sans pour autant être stéréotypée. Après une présentation sous la forme d'une redéfinition tenant compte du contexte, de l'objet de l'article, les auteurs décrivent généralement ses propriétés, en présentant les évolutions potentielles sur l'ensemble de la période, ainsi que le procédé de fabrication. S'ensuivent ensuite, selon l'objet, des considérations sur sa valeur, sa mise en œuvre, ses conditions de vente, ou encore son rôle social et environnemental. L'article se clôt éventuellement par une liste de mots-clés renvoyant à des articles complémentaires, et par une bibliographie de quelques titres de référence. La technique en elle-même n'occupe donc qu'une partie de l'article, mais outre que cette fraction peut être en réalité très développée, son insertion dans une étude d'ensemble du contexte de la «vie» de l'objet donne un éclairage tout à fait bienvenu, même si non technique. Ainsi, ces articles apparaissent comme des condensés, à jour, des connaissances de base permettant une première appréhension globale des enjeux concernant chaque matière traitée.

4 L'article «Métallurgie et sidérurgie » signé Paul Delsalle, traitant d'un sujet d'étude classique en histoire des techniques, est un bon exemple de cette organisation. Développé sur quatre pages, il se divise en deux parties, d'une part les infrastructures de production et d'autre part les produits finis. La première s'ouvre sur une description de la chaîne opératoire de production du fer, du moins par haut-fourneau, en prenant en compte sa diffusion géographique et son évolution dans le temps (avec l'apparition 
de la fonderie à la fin du XVI ${ }^{\mathrm{e}}$ siècle). Les hauts-fourneaux sont décrits brièvement. S'ensuit une analyse de la forge, avec la description d'une partie au moins de son complexe technique: relation à l'environnement, alimentation en énergie, nombre d'ouvriers employés avec la prise en compte chiffrée des variations régionales ainsi que de l'importance des emplois annexes (bûcherons, charbonniers, etc.). Cette partie se termine sur une appréciation de la répartition géographique des forges sur le territoire français, ainsi qu'un point complet sur les « hommes du fer » (spécialisation, salaires, hiérarchie, logement, place sociale). La seconde partie, sur les produits finis, est traitée selon le type de production: armes, marine, horlogerie, ferblanterie, coutellerie, dinanderie, aciérie. Dans tous les cas, les principaux centres de production sont mentionnés, avec des informations chronologiques ou quantitatives, et souvent le statut des artisans ou le cadre légal d'exercice. L'article s'achève sur la description du rôle de ces productions dans l'essor de certains centres urbains à l'époque moderne, avec un phénomène de concentration à la fin du XVIII e siècle annonçant les «usines intégrées" du siècle suivant. Alliant explications générales, données chiffrées et exemples plus précis, cet article réussit donc la gageure de présenter sur quatre pages un panorama complet de ce type d'industrie à l'époque moderne, suffisant pour en comprendre les enjeux dans différents domaines, et fournit en quelque sorte les connaissances indispensables à une première appréhension. La personnalité de l'auteur n'en est pas pour autant gommée, puisque celui-ci, bien qu'ayant travaillé la question en général dans ses publications, s'est également penché particulièrement sur la question de l'environnement, bien appuyée ici. On peut simplement regretter la faiblesse de la bibliographie (outre l'ouvrage de l'auteur sur le sujet, un seul livre de Bertrand Gille datant de plus d'un demi-siècle; l'auteur cite bien Jean-Yves Andrieux dans le cours de l'article, mais ne le redonne pas en bibliographie). D'autre part, il est regrettable que la première partie, sur la chaîne opératoire, se soit exclusivement concentrée sur le fer; l'auteur avait pourtant ouvert l'article en établissant une distinction entre «métallurgie » comme travail des métaux et «sidérurgie » comme travail du fer, mais ne fait finalement référence qu'à cette dernière.

5 Les mêmes remarques peuvent s'appliquer à des articles traitant de sujets bien moins habituels en histoire des techniques. Prenons l'exemple de la "Pêche en eau douce» (la «Pêche en mer» est traitée dans un article séparé), présentée sur une page par Reynald Abad, auteur ayant là encore publié spécifiquement sur ce domaine. L'activité est présentée de manière générale, en prenant en compte son éventuelle illégalité, puis le corps de l'article cette fois encore s'organise en deux parties. Tout d'abord l'expression technique la plus courante de cette activité (la pêcherie) est détaillée, avec ses implantations géographiques privilégiées et les espèces capturées; suit un aperçu de la législation s'y rapportant. La seconde partie se penche sur le particularisme de la pêche en étang en tant qu'«ultime étape d'une pisciculture spécialisée ». On y trouve un descriptif technique de la création et mise en œuvre d'un étang, puis une estimation quantitative et géographique de l'importance du phénomène, et enfin un aperçu des principaux propriétaires de ces installations. La bibliographie se compose de deux ouvrages, dont un de la main de l'auteur de l'article.

6 Malgré l'excellente qualité de la grande majorité des articles, il est tout de même à noter certains moins complets, peut-être du fait d'un plus grand éloignement du sujet par rapport à la spécialité de l'auteur, ou bien d'un choix de se polariser sur une partie de la thématique. Un exemple parlant tient dans la comparaison des articles «Bateaux et navires » et « Construction navale »; tous deux sont signés Michel Vergé-Franceschi, 
bien connu pour ses travaux sur le sujet. Le second article, long et complet, est dans la lignée de ceux commentés précédemment: la technique décrite est plus celle, standardisée, qui s'impose pour les bâtiments militaires à partir de Colbert, mais la construction empirique antérieure ou appliquée à d'autres vaisseaux n'est pas ignorée, et de nombreuses informations sont fournies sur les matériaux employés, les traités, le fonctionnement des chantiers navals, ou encore la sociologie des ouvriers. La bibliographie est bien fournie avec quatre références, même si plus axée sur la sociologie des constructeurs que sur la construction en elle-même. L'article "Bateaux et navires » est par contre beaucoup plus pauvre : après un point sur l'usage des deux termes, l'auteur effectue une description des types de navires en usage à l'époque, et de leurs principaux usages. Si ces informations n'en demeurent pas moins utiles, on aurait pourtant attendu ici un descriptif de la sociologie des marins, des techniques de navigation, ou encore de l'importance des flottes, pour amener l'article au même niveau d'approche totalisante que les précédents. De même, aucune bibliographie n'est fournie, alors même que la somme de Jean Boudriot sur le vaisseau de 74 canons est citée à l'article « Construction navale ».

7 Relevons pour finir un défaut de certains articles, en particulier portant sur l'ameublement lié à la volonté d'y inclure une vision relevant de l'histoire de l'art, tel que présenté dans l'avant-propos. L'article "Table», signé Sophie Mouquin, en est un exemple probant: l'introduction, présentant ce meuble et l'évolution de sa définition depuis le Moyen-Âge, est de bonne facture; mais le reste de l'article s'attache en très grande partie à une description stylistique de la table ainsi que de ses variantes, avec leurs évolutions dans le temps. Bien que ces informations restent essentielles pour l'étude de ce meuble, il manque alors tous les aspects techniques (pour la fabrication), sociologiques (provenance des artisans, logique des choix d'acquisition...), ou encore économiques (provenance et coût des matières premières, d'autant que l'auteur insiste principalement sur les meubles de luxe, prix de vente, zones de diffusion...), qui font justement la richesse de la plupart des articles. Ainsi, pour de nombreux articles en lien avec l'ameublement ou avec des productions luxueuses, l'étude artistique nous semble trop prendre le dessus par rapport au l'approche globalisante qui signe la marque de fabrique de l'ensemble de l'ouvrage.

Si l'on excepte quelques articles plus décevants, qui restent relativement rares, l'ouvrage proposé est donc d'excellente facture et offre les clés d'une première approche complète sur un très grand nombre de sujets liés à la vie matérielle de l'époque moderne. La volonté d'adjoindre une bibliographie aux rubriques (et une autre générale en fin de volume) est louable, mais on peut regretter qu'elle ne soit pas absolument systématique (puisque absente même sur certains articles longs, comme "Pêche en mer " par exemple), et qu'elle privilégie parfois plus des articles de détail que des ouvrages plus généraux et donc plus utiles pour une première approche; il est vrai que l'ouvrage va bientôt fêter son dixième anniversaire et que, l'intérêt actuel pour ces thématiques aidant, certains « incontournables » pour le lecteur de 2016 ont été écrits dans l'intervalle, par exemple celui de Corinne Maitte à propos du verre. Plus gênant peut-être, relevons la quasi-absence d'éléments visuels: quelques pages en couleur sont incluses en milieu d'ouvrage, ainsi que des dessins ou gravures en début de chapitres alphabétiques, mais il ne s'agit là que d'illustrations génériques non commentées. Pour parachever ce caractère «total » des études, il semblerait pourtant indispensable, pour de nombreux articles, de présenter une typologie même sommaire des objets en question, surtout si l'on songe aux navires ou aux tables par exemple. 
Ajoutons à cela un autre manque criant pour un tel ouvrage: la volonté d'inclure d'autres disciplines est de la plus grande importance, même si les résultats peuvent être critiqués, mais comme nous l'avons noté en introduction seule l'histoire de l'art a été convoquée. Or, une étude sur la vie matérielle devrait obligatoirement inclure un aspect archéologique, qu'avait déjà totalement occulté Daniel Roche en son temps. On peut éventuellement admettre que, dans les années 1990, l'archéologie moderniste française n'était pas assez développée pour pouvoir en tirer partie, mais cela est nettement moins vrai pour les années 2000, et n'est plus recevable dans la décennie 2010. L'apport de l'archéologie aurait permis d'étoffer l'argumentaire et la bibliographie de plusieurs articles (par exemple les travaux d'Eric Rieth pour la construction navale), et de fournir un vivier remarquable de documents iconographiques. Ceci mis à part, il s'agit donc là d'un ouvrage remarquable pour acquérir rapidement une vision d'ensemble complète de nombreux éléments techniques incontournables de l'époque moderne.

\section{INDEX}

Mots-clés : histoire des techniques, culture matérielle, pêche, métallurgie, bateau, construction navale, meuble

Keywords : history of technology, material culture, fishing, shipbuilding, metallurgy, furniture Thèmes : Un ouvrage nous $a$ appris

\section{AUTEURS}

\section{CYRIL LACHÈZE}

Cyril Lachèze est doctorant en histoire des techniques sous la direction du professeur AnneFrançoise Garçon, au sein de l'Équipe d'Histoire des techniques de l'Université Paris 1 PanthéonSorbonne (IHMC UMR 8066) et du labex HASTEC. Ses recherches portent sur les relations entre régimes de pensée opératoire, qu'il analyse à travers le cas de la production de terre cuite architecturale du XIII ${ }^{\mathrm{e}}$ au XIX ${ }^{\mathrm{e}}$ siècle. Également formé à l'archéologie moderne et à la musique ancienne, il est par ailleurs éditeur associé au Centre de Musique Baroque de Versailles. Il a été rédacteur en chef adjoint de la revue $e$-Phaïstos. 